\title{
Review of: "Seeing light from a different angle: the effects of diffuse light on the function, structure, and growth of tomato plants"
}

\author{
Mariana Tadey
}

Potential competing interests: The author(s) declared that no potential competing interests exist.

This is a nice work I have only few suggestions regarding the methodology, I am not an expert in this field and I needed more information on the experimental design in order to fully undertand the work done. I attache the manuscript with some comments. 